PROCEEDINGS OF THE

AMERICAN MATHEMATICAL SOCIETY

Volume 125, Number 9, September 1997, Pages 2651-2656

S 0002-9939(97)03878-1

\title{
PEANO PATH DERIVATIVES
}

\author{
HAJRUDIN FEJZIĆ AND DAN RINNE
}

(Communicated by J. Marshall Ash)

\begin{abstract}
In this paper we introduce Peano path derivatives as a natural extension of the notion of path derivatives. We give a sufficient condition on a system of paths to ensure the corresponding Peano path derivative is Baire 1. As consequences, we obtain that unilateral approximate and unilateral $I$ approximate Peano derivatives are Baire one.
\end{abstract}

In studying generalized derivatives, one of the first questions that is addressed is that of Baire classification of the derivative. For example, it is known that ordinary, approximate and Peano derivatives are Baire 1. In [1] the authors introduced path derivatives as a method for unifying approaches to proving properties of various derivatives. Their starting point is the following definition.

Definition 1. Let $x \in R$. A path leading to $x$ is a set $E_{x} \subset R$ such that $x \in E_{x}$ and $x$ is a point of accumulation of $E_{x}$. A system of paths is a collection $E=$ $\left\{E_{x} \mid x \in R\right\}$ such that each $E_{x}$ is a path leading to $x$. For $f: R \rightarrow R$ and a system of paths $E=\left\{E_{x} \mid x \in R\right\}$ we say that $f$ is E-differentiable at $x$ if $\lim _{h \rightarrow 0, x+h \in E_{x}} \frac{f(x+h)-f(x)}{h}$ exists. The limit is called the E-derivative of $f$.

It was shown in [1] that if the system of paths has the property that whenever $x<y$ are sufficiently close the sets $E_{x}$ and $E_{y}$ intersect in the intervals $(2 x-y, x)$ to the left of $x$ and $(y, 2 y-x)$ to the right of $y$, then the $E$-derivative is Baire 1 . The authors called this property the External Intersection Condition (EIC) and, by showing that several generalized derivatives are path derivatives with systems satisfying the EIC, they produced simple proofs that these derivatives are Baire 1.

Due to the bilateral nature of the EIC the analogous results for unilateral generalized derivatives cannot be obtained this way. Also, this condition is not designed to handle higher order generalized derivatives such as Peano derivatives and their generalizations.

Here we introduce Peano path derivatives as a natural extension of the notion of path derivatives. By modifying and generalizing the EIC to this new setting we give a sufficient condition on a system of paths to ensure the corresponding Peano path derivative is Baire 1. As one consequence, we obtain a new and simple proof that approximate Peano derivatives are Baire 1. In fact, by freeing our intersection condition of bilateral restraints, we even show that unilateral approximate and unilateral $\mathcal{I}$-approximate Peano derivatives are Baire 1.

Received by the editors October 25, 1995 and, in revised form, March 20, 1996

1991 Mathematics Subject Classification. Primary 26A24; Secondary 26 A21.

(C)1997 American Mathematical Society 
Definition 2. Let $f: R \rightarrow R$ and let $E=\left\{E_{x} \mid x \in R\right\}$ be a system of paths. We say that $f$ is $n$-times Peano $E$-differentiable at $x$ if there are numbers $f_{1}(x), \ldots, f_{n}(x)$ such that

$$
\lim _{h \rightarrow 0, x+h \in E_{x}} \frac{f(x+h)-f(x)-h f_{1}(x)-\cdots-\frac{h^{n}}{n !} f_{n}(x)}{h^{n}}=0 .
$$

Equivalently

$$
f(x+h)=\sum_{s=0}^{n} \frac{h^{s}}{s !} f_{s}(x)+h^{n} \epsilon_{x}(h)
$$

where $f_{0}(x)=f(x)$ and $\lim _{h \rightarrow 0, x+h \in E_{x}} \epsilon_{x}(h)=0$.

It is easy to check that if $f$ is $n$-times Peano $E$-differentiable at $x$, then it is also $k$-times Peano $E$-differentiable at $x$ for $0 \leq k<n$ with the $k$ th Peano $E$-derivative being $f_{k}(x)$. It follows that the numbers $f_{1}(x), \ldots, f_{n}(x)$ are unique for a fixed path. We call such derivatives Peano path derivatives.

Definition 3. A system of paths satisfies the $n$-uniform intersection condition $(n$ UIC) if there are a positive constant $L$ and a positive function $\delta$ on $R$ so that whenever $0<|y-x|<\min \{\delta(x), \delta(y)\}$ there are $n$ numbers $z_{0}, \ldots, z_{n-1}$ in $E_{x} \cap E_{y}$ with $\min _{i \neq j}\left|z_{i}-z_{j}\right| \geq \frac{|y-x|}{L}$ and $\max _{0 \leq i \leq n-1}\left\{\left|z_{i}-x\right|,\left|z_{i}-y\right|\right\} \leq L|y-x|$.

The essential idea of $n$-UIC is that when $x$ and $y$ are sufficiently close to each other, we can find $n$ numbers in $E_{x} \cap E_{y}$ that are uniformly separated from each other but at the same time uniformly close to both $x$ and $y$ (relative to $|y-x|$ ). This is a modification and generalization of the notion of EIC defined in [1]. Note that a system satisfying the EIC satisfies the 2-UIC with $L=2$.

Theorem 1. Suppose $f$ is $n$-times Peano E-differentiable on $R$ with a system of paths satisfying the $(n+1)$-UIC. For $\epsilon>0$ let

$$
A_{N}=\left\{x \mid \delta(x) \geq \frac{1}{N} \text { and }\left|\epsilon_{x}(h)\right|<\epsilon \text { for all } x+h \in E_{x} \text { and }|h|<\frac{1}{N}\right\} .
$$

For $0 \leq s \leq n$ and $M=4 n !(n+1) !(1+L)^{n(n+1)+n}$, if $x \in \bar{A}_{N}$, then there is $\gamma>0$ such that $y \in \bar{A}_{N}$ and $|x-y|<\gamma$ imply

$$
\left|f_{s}(y)-\sum_{i=s}^{n} \frac{(y-x)^{i-s}}{(i-s) !} f_{i}(x)\right| \leq M \epsilon|y-x|^{n-s} .
$$

Proof. We will use the following identity from Theorem 1.1.17 in [2]. For $x, y, h \in R$ we have

$$
\sum_{s=0}^{n} \frac{h^{s}}{s !}\left(f_{s}(y)-\sum_{i=s}^{n} \frac{(y-x)^{i-s}}{(i-s) !} f_{i}(x)\right)=(y-x+h)^{n} \epsilon_{x}(y-x+h)-h^{n} \epsilon_{y}(h) .
$$

This identity is obtained by writing $f(y+h)$ two ways as follows. First we have $f(y+h)=\sum_{s=0}^{n} \frac{h^{s}}{s !} f_{s}(y)+h^{n} \epsilon_{y}(h)$ by expanding about the point $y$. Then write $y+h$ 
as $y-x+h+x$ and expand about $x$ to get

$$
\begin{aligned}
f(y+h) & =f(y-x+h+x) \\
& =\sum_{i=0}^{n} \frac{(y-x+h)^{i}}{i !} f_{i}(x)+(y-x+h)^{n} \epsilon_{x}(y-x+h) \\
& =\sum_{i=0}^{n} \sum_{s=0}^{i} \frac{(y-x)^{i-s} h^{s}}{s !(i-s) !} f_{i}(x)+(y-x+h)^{n} \epsilon_{x}(y-x+h) \\
& =\sum_{s=0}^{n} \frac{h^{s}}{s !}\left(\sum_{i=s}^{n} \frac{(y-x)^{i-s}}{(i-s) !} f_{i}(x)\right)+(y-x+h)^{n} \epsilon_{x}(y-x+h) .
\end{aligned}
$$

Equating these two expansions gives the desired result.

Now let $x \in \bar{A}_{N}$ and let $L$ be the positive constant associated with the $(n+1)$ UIC. Pick $\min \left\{\delta(x), \frac{1}{N}, \frac{1}{N L}\right\}>\gamma>0$ such that $|h|<L \gamma$ and $x+h \in E_{x}$ imply $\left|\epsilon_{x}(h)\right|<\epsilon$. Let $y \in A_{N}$ with $|y-x|<\gamma$. Since $E$ satisfies the $(n+1)$-UIC condition we can pick $n+1$ points $z_{0}, \ldots, z_{n} \in E_{x} \cap E_{y}$ with $\min _{i \neq j}\left|z_{i}-z_{j}\right| \geq \frac{|y-x|}{L}$ and $\max _{0 \leq i \leq n}\left\{\left|z_{i}-x\right|,\left|z_{i}-y\right|\right\} \leq L|y-x|$. Replacing $h$ with $z_{j}-y$ in (1) we obtain

$$
\begin{aligned}
& \sum_{s=0}^{n} \frac{\left(z_{j}-y\right)^{s}}{s !}\left(f_{s}(y)-\sum_{i=s}^{n} \frac{(y-x)^{i-s}}{(i-s) !} f_{i}(x)\right) \\
& \quad=\left(z_{j}-x\right)^{n} \epsilon_{x}\left(z_{j}-x\right)-\left(z_{j}-y\right)^{n} \epsilon_{y}\left(z_{j}-y\right) \text { for } 0 \leq j \leq n
\end{aligned}
$$

We consider this as the system of linear equations $\left\{\sum_{s=0}^{n} a_{s j} X_{s}=b_{j} \mid 0 \leq j \leq n\right\}$ where $a_{s j}=\left(z_{j}-y\right)^{s}, \quad X_{s}=\frac{1}{s !}\left(f_{s}(y)-\sum_{i=s}^{n} \frac{(y-x)^{i-s}}{(i-s) !} f_{i}(x)\right)$ and $b_{j}=$ $\left(z_{j}-x\right)^{n} \epsilon_{x}\left(z_{j}-x\right)-\left(z_{j}-y\right)^{n} \epsilon_{y}\left(z_{j}-y\right)$.

Let

$$
\Delta=\left[\begin{array}{cccc}
1 & z_{0}-y & \cdots & \left(z_{0}-y\right)^{n} \\
1 & z_{1}-y & \cdots & \left(z_{1}-y\right)^{n} \\
\vdots & \vdots & \ddots & \vdots \\
1 & z_{n}-y & \cdots & \left(z_{n}-y\right)^{n}
\end{array}\right]
$$

and let $\Delta_{s}$ be the matrix obtained by replacing the $s$ th column of $\Delta$ with the values $b_{0}, \ldots, b_{n}$. Then

$$
|\operatorname{det}(\Delta)|=\left|\prod_{i>j}\left(z_{i}-z_{j}\right)\right| \geq\left(\frac{|y-x|}{L}\right)^{\frac{n(n+1)}{2}}>0 .
$$

In the expansion of $\operatorname{det}\left(\Delta_{s}\right)$ about the $s$ th column, each minor is the sum of $n$ ! terms of the form $( \pm) \prod_{j \neq s}\left(z_{j}-y\right)^{k_{j}}$ where $\sum_{j \neq s} k_{j}=\frac{n(n+1)}{2}-s$. Therefore

$$
\left|\operatorname{det}\left(\Delta_{s}\right)\right| \leq \sum_{j=0}^{n}\left|b_{j}\right| n ! \max _{0 \leq i \leq n}\left|z_{i}-y\right|^{\frac{n(n+1)}{2}-s}
$$


Since $\left|z_{j}-x\right|<L \gamma$ and $\left|z_{j}-y\right|<\delta(y),\left|b_{j}\right| \leq 2 \epsilon L^{n}|y-x|^{n}$. It follows that

$$
\begin{aligned}
\left|\operatorname{det}\left(\Delta_{s}\right)\right| & \leq \sum_{j=0}^{n} 2 \epsilon L^{n}|y-x|^{n} n ! L^{\frac{n(n+1)}{2}-s}|y-x|^{\frac{n(n+1)}{2}-s} \\
& =(n+1) ! 2 \epsilon L^{\frac{n(n+1)}{2}+n-s}|y-x|^{\frac{n(n+1)}{2}+n-s} .
\end{aligned}
$$

By Cramer's Rule

$$
\begin{aligned}
& \left|\frac{1}{s !}\left(f_{s}(y)-\sum_{i=s}^{n} \frac{(y-x)^{i-s}}{(i-s) !} f_{i}(x)\right)\right|=\left|\frac{\operatorname{det}\left(\Delta_{s}\right)}{\operatorname{det}(\Delta)}\right| \\
& \quad \leq(n+1) ! 2 \epsilon L^{n(n+1)+n-s}|y-x|^{n-s} \leq \frac{M}{2 s !} \epsilon|y-x|^{n-s}
\end{aligned}
$$

where $M=4 n !(n+1) !(1+L)^{n(n+1)+n}$.

Finally if $y \in \bar{A}_{N}$ with $|y-x|<\gamma$, pick a sequence $\left\{y_{m}\right\} \subset A_{N}$ converging to $y$, and such that $\left|y_{m}-x\right|<\gamma$. Replacing $y$ with $y_{m}$, the calculation above shows that $\left|f_{s}\left(y_{m}\right)-\sum_{i=s}^{n} \frac{\left(y_{m}-x\right)^{i-s}}{(i-s) !} f_{i}(x)\right| \leq \frac{M}{2} \epsilon\left|y_{m}-x\right|^{n-s}$ for $0 \leq s<n$ and $\left|f_{n}\left(y_{m}\right)-f_{n}(x)\right| \leq \frac{M}{2} \epsilon$. Replacing $x$ with $y$, the same formulas yield $\lim _{m \rightarrow \infty} f_{s}\left(y_{m}\right)=$ $f_{s}(y)$ for $0 \leq s<n$ and $\limsup \left|f_{n}\left(y_{m}\right)-f_{n}(y)\right| \leq \frac{M}{2} \epsilon$. Letting $m \rightarrow \infty$ we obtain the desired inequality for $0 \leq s<n$, while for $s=n$ we have

$$
\begin{aligned}
\left|f_{n}(y)-f_{n}(x)\right| & \leq \limsup \left|f_{n}\left(y_{m}\right)-f_{n}(x)\right|+\lim \sup \left|f_{n}\left(y_{m}\right)-f_{n}(y)\right| \\
& \leq \frac{M}{2} \epsilon+\frac{M}{2} \epsilon \leq M \epsilon . \quad \square
\end{aligned}
$$

Remark 1 . From the inequality demonstrated in Theorem 1 we immediately see that for $0 \leq s<n$ the $s$ th order Peano $E$-derivatives of $f$ restricted to $\bar{A}_{N}$ are continuous and the oscillation of $f_{n}$ restricted to $\bar{A}_{N}$ is bounded by $M \epsilon$.

Corollary 2. Suppose $f$ is n-times Peano E-differentiable on $R$ with a system of paths satisfying the $(n+1)$-UIC. Then $f_{s}$ is Baire ${ }^{*} 1$ for $0 \leq s<n$ and $f_{n}$ is Baire 1 .

Proof. Let $C$ be a closed subset of $R, \epsilon>0$ and $A_{N}$ as in Theorem 1. Since $\bigcup_{N=1}^{\infty} \bar{A}_{N}=R$, by the Baire Category Theorem there are a closed interval $I_{\epsilon} \subset R$ and an integer $N$ such that $C \cap I_{\epsilon}$ is nonempty and contained in $\bar{A}_{N}$. By Theorem 1, for $0 \leq s<n$ the $s$ th order Peano $E$-derivatives of $f$ restricted to $\bar{A}_{N}$ are continuous. Therefore, the restrictions of these derivatives to the set $C \cap I_{\epsilon}$ are also continuous. We also have the oscillation of $\left.f_{n}\right|_{C \cap I_{\epsilon}}$ less than or equal to $M \epsilon$. By picking a sequence $\left\{\epsilon_{i}\right\}$ decreasing to zero we obtain a nested sequence of corresponding intervals $\left\{I_{\epsilon_{i}}\right\}$ with the oscillation of $\left.f_{n}\right|_{C \cap I_{\epsilon_{i}}}$ less than or equal to $M \epsilon_{i}$. Thus $\left.f_{n}\right|_{C}$ is continuous at each $x \in C \cap\left(\cap I_{\epsilon_{i}}\right)$.

It is easy to see that right approximate Peano derivatives are Peano path derivatives using, as a path at each $x$, a corresponding set $E_{x}$ with right density one at $x$ for which

$$
\lim _{h \rightarrow 0^{+}, x+h \in E_{x}} \frac{f(x+h)-f(x)-h f_{1}(x)-\cdots-\frac{h^{n}}{n !} f_{n}(x)}{h^{n}}=0 .
$$


Similarly the left approximate Peano derivatives are Peano path derivatives.

Corollary 3. Suppose $f$ is $n$-times right (left) approximate Peano differentiable on $R$. Then $f_{s}$ is Baire* 1 for $0 \leq s<n$ and $f_{n}$ is Baire 1 .

Proof. Since right approximate Peano derivatives are Peano path derivatives, it suffices to show that the corresponding system of paths $E=\left\{E_{x}\right\}$, where $E_{x}$ has right density one at $x$, satisfies the $(n+1)$-UIC. For each $x \in R$ pick $\delta(x)$ so that $0<h<\delta(x)$ implies

$$
\min \left\{\frac{\lambda\left(E_{x} \cap(x, x+h)\right.}{h}, \frac{\lambda\left(E_{x} \cap(x+h, x+2 h)\right.}{h}\right\}>\frac{2 n+2.5}{2 n+3} .
$$

Suppose $x<y$ and $y-x<\min \{\delta(x), \delta(y)\}$. Since $\frac{\lambda\left(E_{x} \cap E_{y} \cap(y, 2 y-x)\right)}{y-x}>$ $\frac{2 n+2}{2 n+3}$, if we divide the interval $(y, 2 y-x)$ into $2 n+3$ subintervals of equal length $\left\{I_{k}\right\}_{k=0}^{2 n+2}$, each $E_{x} \cap E_{y} \cap I_{k} \neq \varnothing$. Pick $z_{i} \in E_{x} \cap E_{y} \cap I_{2 i+1}$. Then $E$ satisfies the $(n+1)$-UIC with $L=2 n+3$.

The category analog of the approximate Peano derivative is called the $\mathcal{I}$-approximate Peano derivative and can similarly be thought of as a Peano path derivative. Specifically, the right $\mathcal{I}$-approximate Peano derivatives are Peano path derivatives using, as a path at each $x$, a corresponding set $E_{x}$ which has $x$ as a right $\mathcal{I}$-density point for which

$$
\lim _{h \rightarrow 0^{+}, x+h \in E_{x}} \frac{f(x+h)-f(x)-h f_{1}(x)-\cdots-\frac{h^{n}}{n !} f_{n}(x)}{h^{n}}=0 .
$$

Similarly the left $\mathcal{I}$-approximate Peano derivatives are Peano path derivatives.

We will use the following lemma of W. Poreda, E. Wagner-Bojakowska, and W. Wilczyński [4] and theorem of E. Lazarow [3] (the author proved in this work that $\mathcal{I}$-approximate derivatives are Baire 1 ) to prove Corollary 6 below.

Lemma 4. If 0 is an $\mathcal{I}$-density point of the set $A$, then for every natural number $n$, there exists a real number $\delta>0$ such that, for each $h \in(0, \delta)$ and for each natural number $k$ with $-n \leq k \leq n-1$ we have $A \cap\left[\frac{k}{n} h, \frac{k+1}{n} h\right] \neq \phi$.

Note that in this lemma we may assume the intersection is second category since we may assume that if $A \cap I \neq \phi$ for any interval $I$, then $A \cap I$ is of second category..

Theorem 5. The point 0 is an $\mathcal{I}$-dispersion point of the open set $G$ if and only if for every natural number $n$ there exists a natural number $k$ and a real number $\delta>0$ such that, for each $h \in(0, \delta)$ and for each $i \in\{1, \ldots, n\}$ there exist two natural numbers $j_{r}, j_{l} \in\{1, \ldots, k\}$ such that $G \cap\left(\left(\frac{i-1}{n}+\frac{j_{r}-1}{n k}\right) h,\left(\frac{i-1}{n}+\frac{j_{r}}{n k}\right) h\right)=\phi$ and $G \cap\left(-\left(\frac{i}{n}+\frac{j_{l}}{n k}\right) h,-\left(\frac{i}{n}+\frac{j_{l}-1}{n k}\right) h\right)=\phi$.

If $A$ is a set with the Baire Property then both $A$ and its complement differ from open sets by first category sets. Thus Theorem 5 can be used to say that if 0 is an $\mathcal{I}$-density point of the set $A$ then $A$ is residual in the two subintervals specified in the theorem since the complement of $A$ is first category in those subintervals. The obvious unilateral variants of the lemma and theorem above also hold.

Corollary 6. Suppose $f$ is $n$-times right (left) $\mathcal{I}$-approximate Peano differentiable on $R$. Then $f_{s}$ is Baire* 1 for $0 \leq s<n$ and $f_{n}$ is Baire 1 . 
Proof. Since right $\mathcal{I}$-approximate Peano derivatives are Peano path derivatives, it suffices to show that the corresponding system of paths $E=\left\{E_{x}\right\}$ satisfies the $(n+1)$-UIC. By Theorem 5 for each $x$ there are a $k_{x}$ and a $\delta_{1}(x)>0$ so that $0<h<$ $\delta_{1}(x)$ implies if we split $(x, x+h)$ into $2 n+3$ equal intervals and further subdivide each of those into $2^{k_{x}}$ subintervals, then in each of the $2 n+3$ intervals to the right of $x, E_{x}$ is residual in one of the $2^{k_{x}}$ subintervals. Now apply Lemma 4 using $(2 n+3) 2^{k_{x}}$ in place of $n$ to get a corresponding $\delta_{2}(x)$. Let $\delta(x)=\frac{1}{2 n+3} \min \left(\delta_{1}(x), \delta_{2}(x)\right)$. Now suppose $x<y$ and $y-x<\min (\delta(x), \delta(y))$. We first consider the case $k_{x} \leq k_{y}$. Using $h=(2 n+3)(y-x)$ we have that if $(x, x+h)$ is divided into $2 n+3$ intervals, each of these has $E_{x}$ residual in one of its $2^{k_{x}}$ subintervals (by Theorem 5 ). In each of the $2 n+2$ intervals to the right of $y$, each of its $2^{k_{y}} \geq 2^{k_{x}}$ subintervals hits $E_{y}$ in a second category set (by Lemma 4). Thus $E_{x} \cap E_{y} \cap\left(y+\frac{2 j}{2 n+3} h, y+\frac{2 j+1}{2 n+3} h\right) \neq \phi$ for $0 \leq j \leq n$ and we have the $(n+1)$-UIC with $L=2 n+3$. A similar argument applies if $k_{x}>k_{y}$. Again using $h=(2 n+3)(y-x)$ we have that if $(y, y+h)$ is divided into $2 n+3$ intervals, each of these has $E_{y}$ residual in one of its $2^{k_{y}}$ subintervals. In each of the $2 n+2$ intervals to the right of $y$, each of its $2^{k_{x}}>2^{k_{y}}$ subintervals hits $E_{x}$ in a second category set and we obtain the $(n+1)$-UIC.

\section{REFERENCES}

[1] A. M. Bruckner, R. J. O'Malley and B. S. Thomson, Path Derivatives: A Unified View of Certain Generalized Derivatives, Trans. Amer Math. Soc. Vol. 2831 (1984), 97-125. MR 86d:26007

[2] H. Fejzić, The Peano Derivatives, Ph.D. Dissertation, Michigan State University (1992).

[3] E. Lazarow, On the Baire Class of $\mathcal{I}$-approximate Derivatives, Proc. Amer. Math. Soc. Vol. 1004 (1987), 669-674. MR 88h:26001

[4] W. Poreda, E. Wagner-Bojakowska, and W. Wilczyński, A Category Analogue of the Density Topology, Fund. Math. Vol. 128 (1985), 167-173. MR 87b:54034

Department of Mathematics, California State University, San Bernardino, CaliforNIA 92407

E-mail address: hfejzic@wiley.csusb.edu

E-mail address: drinne@wiley.csusb.edu 